\title{
Guidance for Healthcare Providers Managing COVID-19 in Rural and Underserved Areas
}

\author{
Sunil Sharma ${ }^{1}$ (D) James Cain ${ }^{2} \cdot$ Ankit Sakhuja $^{3} \cdot$ Gregory Schaefer $^{4} \cdot$ Troy Krupica $^{5} \cdot$ Arif Sarwari $^{6}$
}

Received: 31 May 2020 /Revised: 29 June 2020 / Accepted: 5 July 2020 / Published online: 10 July 2020

(C) W. Montague Cobb-NMA Health Institute 2020

\begin{abstract}
Coronavirus disease 2019 (COVID-19), which is caused by severe acute respiratory syndrome coronavirus 2 (SARS-CoV-2), has ravaged many urban and high-density areas in the USA. However, rural areas (despite their low population density) may be especially vulnerable to poor outcomes from COVID-19, owing to limited healthcare infrastructure, long distances to advanced health care, and population characteristics (e.g., high tobacco use, hypertension, obesity, older age). A panel of experts who are actively engaged in treating and managing COVID-19 at a rural academic center was convened to address this topic. In this commentary, we provide readers with some specific issues faced by rural healthcare providers and offer guidance in overcoming these challenges. This guidance includes alternative ventilator strategies, personal protective equipment (PPE), and common therapeutic options.
\end{abstract}

Keywords COVID-19 $\cdot$ Rural health $\cdot$ Pandemic $\cdot$ Ventilation strategies

\section{Introduction}

Coronavirus disease 2019 (COVID-19), caused by severe acute respiratory syndrome coronavirus 2 (SARS-CoV-2), is believed to have emerged in Wuhan, China, in December 2019 [1]. Thereafter, it spread worldwide. Declared a pandemic by the World Health Organization (WHO) on March 11, 2020, COVID-19 has infected individuals in every US state, ravaging many urban and high-density areas. Rural areas,

Sunil Sharma

Sunil.Sharma@hsc.wvu.edu

1 Division of Pulmonary, Critical Care \& Sleep Medicine, Department of Medicine, West Virginia University, PO Box 9156, Morgantown, WV 26506, USA

2 Department of Anesthesiology, West Virginia University, One Medical Center, Morgantown, WV 26506, USA

3 Division of Cardiovascular Critical Care, Department of Cardiovascular and Thoracic Surgery, West Virginia University, PO Box 8500, Morgantown, WV 26506, USA

4 Department of Surgery, West Virginia University, One Medical Center, Morgantown, WV 26506, USA

5 Division of Hospital Medicine, West Virginia University, PO Box 9156, Morgantown, WV 26506, USA

6 Department of Medicine, West Virginia University, PO Box 9156, Morgantown, WV 26506, USA despite having a low population density, may be especially vulnerable to poor outcomes from COVID-19 owing to limited healthcare infrastructure, long distances to advanced health care, and population characteristics (e.g., tobacco use, hypertension, obesity, older age) [2]. Since the report of the first rural case on February 20, 2020, in Humboldt County, a rural county in Northern California, COVID-19 has reached almost two-thirds of all rural counties [3]. Rural areas have limited availability of Intensive Care Unit (ICU) beds, ventilators, and access to novel medications through clinical trials. To meet such challenges, West Virginia University Medicine formed an Expert Scientific Panel to address COVID-19 issues particularly relevant to rural hospital care. The Panel shares guidance on ventilation strategies, healthcare protective equipment medications, and screening below.

\section{Alternative Modes of Ventilation in View of Limited Ventilator Availability}

COVID-19 acute respiratory distress syndrome (ARDS) patients may require significantly longer ventilator support than nonCOVID ARDS patients. These prolonged ventilation needs may occupy and deplete the main ICU ventilator supply. Considering the pervasive shortage of ICU beds/ventilators in rural areas, alternative means of ventilatory support need to be explored and utilized. Most medical facilities have a supply of 
ventilatory assist systems used predominantly for non-invasive ventilators (e.g., V-60's, Trilogy, Astrals and Bilevel devices) which can be easily converted for use as invasive ventilators. While lacking advanced modes available on ICU ventilators, they are suitable for use in patients with milder types of respiratory failures (e.g., COPD exacerbations, acute hypercapnic respiratory failure). They may also be useful for ventilated ICU patients being weaned and on minimal support [4]. Additionally, patients who own home Bilevel devices can be modified into ventilators. The Panel recommends that healthcare providers and respiratory therapists become familiar with nontraditional ICU ventilators like home ventilators (home ventilators, Bilevel devices, V-60 ventilators) and how to convert these non-invasive ventilators into invasive ventilators. The circuit instead of connecting to the face mask can be connected to the endotracheal tube (ET) with a disposable expiratory port (DEP) and HEPA filter attachments (Fig. 1). The preferable ventilator settings while using these ventilators would be BiPAP s/t. However, pressure control ventilation (PCV) can also be used. These modes of ventilation are quite similar except for the inspiratory time (I-time) in BiPAP s/t being variable and patientdriven while being fixed in PCV. We prefer BiPAP $s / t$ mode for this reason as it may be more comfortable to the patient who may require less sedation. An inline suction catheter or a nebulizer pump can also be easily connected.

Another significant resource are anesthesia machines. These can function as ventilators, with sophisticate modes available on many of the newest generation devices [5]. Furthermore, since only urgent and emergent surgeries have been performed during the pandemic, there are many underutilized anesthesia machines as well as anesthesiologists, nurse anesthetists, and anesthesiology assistants available to provide care [5].

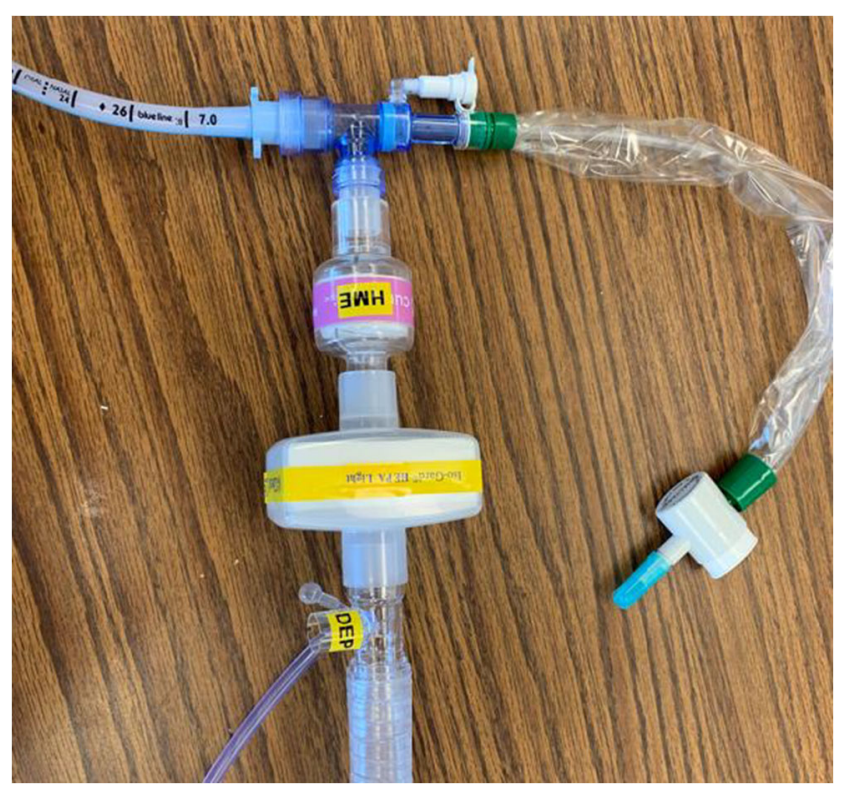

Fig. 1 Humidifier, HEPA filter, and DEP port connected in sequence from an ET tube to the circuit
Role of Novel PPE in View of a Nationwide Shortage of Personal Protective Equipment

In light of the high risk of transmission of the virus to healthcare workers and patients and an alarming shortage of personal protective equipment nationwide, we strongly recommend the use of novel protective equipment (PPE). We have noted initiatives by many rural healthcare systems in this regard. We recommend collaboration with local expertise (e.g., collaboration between the Medical School and the Biomedical Engineering Department) to explore this area of critical need. In the interim, the Panel suggests the utilization of anesthesia masks with HEPA filters as one such stop gap option (Fig. 2). It is a relatively simple technique with readily available resources considering the low utilization of masks owing to the significant reduction of surgeries during this crisis.

While novel masks may not be Occupational Safety and Health Administration (OSHA) compliant, using innovative and scientifically sound methods to create a PPE is preferred to insufficient or no protection [6]. In addition to anesthesia mask repurposing, other options include using the sterile wrapping material from surgical instruments as raw material to sew masks or mass produce N95s and developing face shields using 3-D printers [7].

\section{Use Clear Drapes or Intubation Boxes for High-Risk Airway Procedures on COVID-19 Patients}

Co-infection of healthcare workers (HCW) in hot spots has been reported to be high and most likely attributable to the aerosolization of viral particles. At highest risk are those in closest proximity to the aerodigestive system. An extra barrier, such as a clear drape which can be easily obtained and

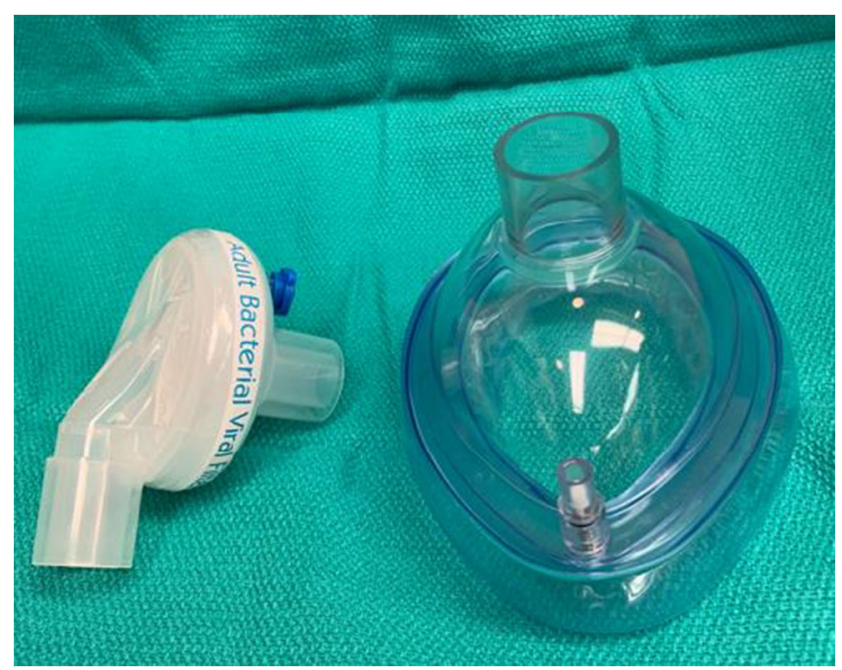

Fig. 2 Anesthesia mask along with attachable HEPA filter 
disposed post-procedure, or a reusable Plexiglas intubation box is consistent with best practices [8]. Plastic drapes may be more easily available in rural hospitals and would complement any local PPE measures. We recommend the use of clear drapes or Plexiglas ${ }^{\circledR}$ intubation boxes for all airway-linked procedures (intubation, extubation, cuff leak test, bronchoscopy, tracheal aspirates) to reduce the risk of aerosolization and HCW exposure. Clear plastic drapes are inexpensive and easily accessible in rural medical settings.

\section{Universal Screening in Rural Areas}

During the earlier stages of a pandemic, there typically is an acute shortage of testing devices (e.g., nasopharyngeal swabs, reagents, and test kits), making it neither practical nor beneficial to promote universal screening in rural areas. However, as soon as possible, liberal screening of HCW should be promoted. This should start with hospital employees and has two primary purposes: (1) to prevent inadvertent transmission to the vulnerable hospital population (both patients and coworkers) and (2) to provide an estimate of the local penetrance of COVID-19. The other vulnerable population is rural nursing home residents. Rural elders are at a higher risk of nursing home admission than their urban counterparts [9]. In nursing homes, healthcare employees are the main initiators of an outbreak. Liberal and regular screening of this group will serve to keep this population protected.

\section{Convalescent Plasma as a Therapeutic Option for COVID-19}

Convalescent plasma has been used in life-threatening medical conditions in the past including SARS-CoV-1 showing reduction in mortality [10]. Animal models and human experience in the treatment of influenza pneumonia and ARDS suggest it may be an effective therapeutic modality [11]. In the case of COVID-19, viral neutralization is the anticipated mechanism of action by which passive antibody therapy would mediate protection. To be most effective, this therapy is recommended to be instituted early in the course of disease [10]. Additionally, given the increasing number of COVID-19 cases in rural areas, there is an expanded pool of potential donors and hence may be more readily available. The Federal Food and Drug Agency (FDA) has made possible an expedited Investigational New Drug process for plasma use in the fight against COVID-19 for emergency and lifesaving situations.

We recommend consideration of convalescent plasma for COVID-19 treatment within the context of a clinical trial. If rural facilities are unable to participate in a national clinical trial, FDA has opened a pathway for Investigational New
Drug (IND) applications to allow expanded access to convalescent plasma [12].

\section{Remdesivir}

Remdesivir is an anti-viral drug which is an adenosine analog and mimics as a building block of RNA to stops viral replication. It was unsuccessfully used in the past for Ebola virus treatment. A randomized control trial performed in China did not show any benefit in outcome [13]; however, the trial was prematurely terminated owing to poor enrollment. A randomized control trial (known as the Adaptive COVID-19 Treatment Trial, or ACTT) [14], sponsored by the National Institute of Allergy and Infectious Diseases (NIAID), is currently underway. The Data and Safety Monitoring Board (DSMB) recently shared its preliminary findings which showed median time to recovery of 11 days for patients treated with remdesivir versus patients receiving placebo. The results also show a survival benefit in patients receiving remdesivir versus those receiving placebo ( $8 \%$ versus $11.6 \%$ ). Based on these initial results, the FDA issued an emergency use authorization (EUA) allowing remdesivir to be used for critically ill hospitalized patients. The drug is now being allocated to hospitals/regions across the country based on the principle of maximizing the number of lives saved.

We recommend that remdesivir be considered for treatment of critically ill patients in the ICU settings within the guidelines established by the trial. At our Institution, a mandatory infectious disease consult is required before initiating remdesivir.

\section{Hydroxychloroquine and Azithromycin for COVID-19}

The data supporting hydroxychloroquine for COVID-19 is weak and largely anecdotal. The data supporting hydroxychloroquine in critically ill COVID-19 patients is even less supportive [15]. The strongest evidence comes from a small study of 20 patients at a single hospital in France [16]. Of these patients, only 6 received combination therapy (addition of azithromycin). Since then, several studies including a randomized controlled trial [17] have shown either no benefit or in some cases higher adverse events [18]. The FDA has recently cautioned against the use of hydroxychloroquine. Physicians must also be cognizant that utilizing hydroxychloroquine for COVID-19 patients in rural areas may result in an acute shortage for patients depending upon this drug to treat lupus and rheumatoid arthritis. We recommend against the use of this combination until more favorable data is available. AS of June 15, 2020, the Food and Drug 
Administration (FDA) has revoked emergency use authorization (EUA) for the use of hydroxychloroquine for the treatment of COVID-19 outside the setting of a clinical trial.

\section{Role of Tocilizumab in the Treatment of COVID-19}

There is a paucity of data and unclear indication to use tocilizumab for COVID-19. However, a cytokine profile resembling secondary hemophagocytic lymphohistiocytosis (sHLH) is associated with COVID-19 disease severity, characterized by increased interleukin (IL)-2, IL-6, IL-7, granulocyte-colony stimulating factor, interferon- $\gamma$ inducible protein 10 , monocyte chemoattractant protein 1 , macrophage inflammatory protein $1-\alpha$, and tumor necrosis factor- $\alpha$ [19]. Predictors of fatality from a recent retrospective, multi-center study of 150 confirmed COVID-19 cases in Wuhan, China, included elevated ferritin (mean $1297.6 \mathrm{ng} / \mathrm{ml}$ in nonsurvivors vs $614.0 \mathrm{ng} / \mathrm{ml}$ in survivors; $p<0.001$ ) and IL-6 $(p<0 \cdot 0001)$, suggesting that mortality might be attributable to virally driven hyperinflammation [20]. Tocilizumab's utility in "cytokine release syndrome" (FDA-approved) may also suggest potential benefit. However, these benefits need to be balanced against availability of this drug in rural areas. We suggest its use as investigational with appropriate IRB oversight.

\section{Conclusion}

During the COVID-19 crisis, it is critical that rural healthcare systems are prepared and informed of best practices. To meet such challenges, West Virginia University Medicine formed an Expert Scientific Panel to address COVID-19 issues and management in rural settings. Medicare defines rural areas as all counties outside Metropolitan Statistical Area (MSA). The Panel recognizes the limitations given the dearth of validated literature for COVID-19 and makes its recommendations based upon (1) scientific foundations from studies of similar disease processes, (2) anecdotal COVID-19 information, and (3) scientifically sound and novel use of existing products, including de novo product development. With these considerations, the panel recommends (1) the repurposing of equipment to offset ventilator shortages, (2) using alternative, lowcost forms of PPE, and (3) the targeted use of certain drugs. While the above recommendations are intended to facilitate patient care in low-resource environments, clinicians and hospital administrators are advised to consult appropriate medical oversight committees and device manufactures before implementing alterations of medical equipment (to avoid any unintended safety and performance issues).
Author Contributions Conceptualization was by Sunil Sharma, James Cain, Ankit Sakhuja, Gregory Schaefer, Troy Krupica, and Arif Sarwari. Writing and original draft preparation was by Sunil Sharma. Resources were provided by Sunil Sharma, James Cain, Ankit Sakhuja, Gregory Schaefer, Troy Krupica, and Arif Sarwari. Review and editing were by Sunil Sharma, James Cain, Ankit Sakhuja, Gregory Schaefer, Troy Krupica, and Arif Sarwari.

\section{Compliance with Ethical Standards}

Conflict of Interest The authors declare that they have no conflict of interest.

Disclaimer The views expressed in this manuscript do not necessarily reflect the position or policy of West Virginia University, the State of West Virginia, or the United States. Government.

\section{References}

1. Zhou P, Yang XL, Wang XG, Hu B, Zhang L, Zhang W, et al. A pneumonia outbreak associated with a new coronavirus of probable bat origin. Nature. 2020;579(7798):270-3.

2. Ranscombe P. Rural areas at risk during COVID-19 pandemic. Lancet Infect Dis. 2020;20(5):545.

3. Healy J, Tavernise S., Gebeloff R, Cai W. Coronavirus was slow to spread to rural America. Not anymore. The New York Times Apr 8, 2020. 2020

4. Mittal A, Forte M, Leonard R, Sangani R, Sharma S. Refractory acute respiratory distress syndrome secondary to COVID-19 successfully extubated to average volume-assured pressure support non-invasive ventilator. Cureus. 2020;12(4):e7849.

5. APSF/ASA Guidance on Purposing Anesthesia Machines as ICU Ventilators. https://www.asahq.org/in-the-spotlight/coronaviruscovid-19-information/purposing-anesthesia-machines-forventilators (May 22, 2020),

6. Garibaldi BT, Ruparelia C, Shaw-Saliba K, Sauer LM, Maragakis LL, Glancey M, et al. A novel personal protective equipment coverall was rated higher than standard Ebola virus personal protective equipment in terms of comfort, mobility and perception of safety when tested by health care workers in Liberia and in a United States biocontainment unit. Am J Infect Control. 2019;47(3):298-304.

7. Amin D, Nguyen N, Roser SM, Abramowicz S. 3D printing of face shields during COVID-19 pandemic: a technical note. J Oral Maxillofac Surg. 2020.

8. Canelli R, Connor CW, Gonzalez M, Nozari A, Ortega R. Barrier enclosure during endotracheal intubation. N Engl J Med. 2020;382(20):1957-8.

9. Coward RT, Netzer JK, Mullens RA. Residential differences in the incidence of nursing home admissions across a six-year period. J Gerontol B Psychol Sci Soc Sci. 1996;51(5):S258-67.

10. Mair-Jenkins J, Saavedra-Campos M, Baillie JK, Cleary P, Khaw FM, Lim WS, et al. The effectiveness of convalescent plasma and hyperimmune immunoglobulin for the treatment of severe acute respiratory infections of viral etiology: a systematic review and exploratory meta-analysis. J Infect Dis. 2015;211(1):80-90.

11. Mozdzanowska K, Furchner M, Washko G, Mozdzanowski J, Gerhard W. A pulmonary influenza virus infection in SCID mice can be cured by treatment with hemagglutinin-specific antibodies that display very low virus-neutralizing activity in vitro. J Virol. 1997;71(6):4347-55.

12. Administration, U. F. D. Recommendations for Investigational COVID-19 Convalescent Plasma. https://www.fda.gov/vaccinesblood-biologics/investigational-new-drug-ind-or-device- 
exemption-ide-process-cber/recommendations-investigationalcovid-19-convalescent-plasma (May 23, 2020),

13. Wang Y, Zhang D, Du G, Du R, Zhao J, Jin Y, et al. Remdesivir in adults with severe COVID-19: a randomised, double-blind, placebo-controlled, multicentre trial. Lancet. 2020;395(10236):1569-78.

14. Beigel JH, Tomashek KM, Dodd LE, Mehta AK, Zingman BS, Kalil AC, et al. Remdesivir for the treatment of COVID-19 - preliminary report. N Engl J Med. 2020.

15. Molina JM, Delaugerre C, Le Goff J, Mela-Lima B, Ponscarme D, Goldwirt L, et al. No evidence of rapid antiviral clearance or clinical benefit with the combination of hydroxychloroquine and azithromycin in patients with severe COVID-19 infection. Med Mal Infect. 2020;50(4):384.

16. Gautret P, Lagier JC, Parola P, Hoang VT, Meddeb L, Mailhe M, et al. Hydroxychloroquine and azithromycin as a treatment of COVID-19: results of an open-label non-randomized clinical trial. Int J Antimicrob Agents. 2020;105949.

17. Tang W, Cao Z, Han M, Wang Z, Chen J, Sun W, et al. Hydroxychloroquine in patients with mainly mild to moderate coronavirus disease 2019: open label, randomised controlled trial. BMJ. 2020;369:m1849.

18. Chorin E, Wadhwani L, Magnani S, Dai M, Shulman E, NadeauRouthier C, et al. QT interval prolongation and torsade de pointes in patients with COVID-19 treated with hydroxychloroquine/ azithromycin. Heart Rhythm. 2020.

19. Mehta P, McAuley DF, Brown M, Sanchez E, Tattersall RS, Manson JJ, et al. COVID-19: consider cytokine storm syndromes and immunosuppression. Lancet. 2020;395(10229):1033-4.

20. Ruan Q, Yang K, Wang W, Jiang L, Song J. Clinical predictors of mortality due to COVID-19 based on an analysis of data of 150 patients from Wuhan, China. Intensive Care Med. 2020;46(5):8468.

Publisher's Note Springer Nature remains neutral with regard to jurisdictional claims in published maps and institutional affiliations. 